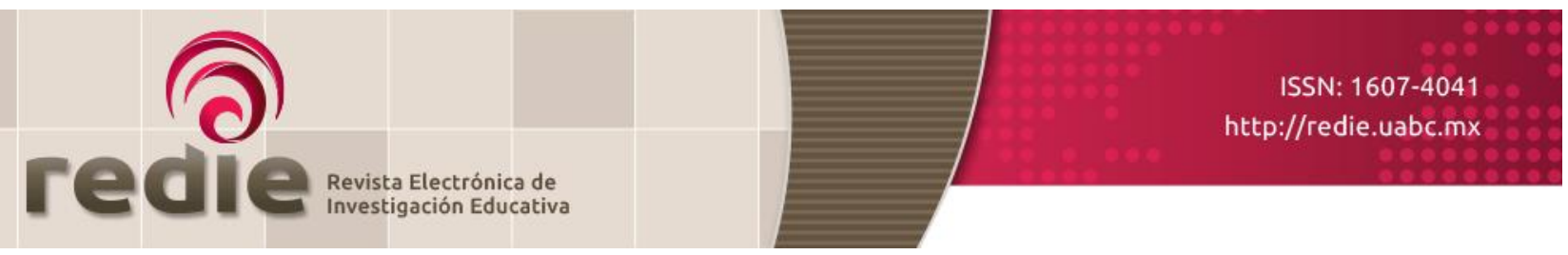

Vol. 21, 2019/e32

\title{
Autoeficacia académica en estudiantes de Psicología de una universidad de Lima
}

\section{Academic Self-Efficacy in Psychology Students from a University in Lima}

Sergio Dominguez-Lara $\left(^{*}\right)$ sdominguezmpcs@gmail.com

Manuel Fernández-Arata $(*)$ mfernandeza1@usmp.pe

(*) Universidad de San Martín de Porres

(Recibido: 2 de junio de 2017; Aceptado para su publicación: 28 de noviembre de 2017)

Cómo citar: Dominguez-Lara, S. y Fernández-Arata, M. (2019). Autoeficacia académica en estudiantes de Psicología de una universidad de Lima. Revista Electrónica de Investigación Educativa, 21, e32, 1-13. doi:10.24320/redie.2019.21.e32.2014

\section{Resumen}

El objetivo de este estudio descriptivo fue conocer la prevalencia de baja Autoeficacia Académica (AA) en estudiantes de una universidad privada de Lima (Perú). Participaron 730 estudiantes de Psicología (76.6\% mujeres) de entre 16 y 47 años $(M=20.72)$ de 10. a 5o. año, quienes fueron evaluados con la Escala de Autoeficacia Percibida Específica de Situaciones Académicas. Los análisis preliminares estuvieron basados en métodos analítico-factoriales orientados a explorar la equivalencia de la medida de AA de acuerdo al sexo y año de estudios. Los resultados indican que el 17.4\% presenta baja AA, el 61.2\% AA promedio y un $21.4 \%$ reporta altos niveles de AA. Los hombres reportan mayor AA que las mujeres y los estudiantes de 5o. año indican más AA que los de 10.

Palabras clave: Autoeficacia académica, autoeficacia percibida, estudiante universitario.

\section{Abstract}

The aim of this descriptive study was to know the prevalence of academic sef-efficacy (ASE) in college students of a private university of in Lima. Participants were 730 psychology students $(76.6 \%$ women) with ages between 16 and 47 years old $(M=20.72)$ from the 1 st to the last year of college. As instrument we used the Scale of Specific Perceived Self-Efficacy of Academic Situations. The preliminary analysis was based on factor analysis, having as main objective to explore the measurement equivalence regarding gender and year of study. Results showed that the $17.4 \%$ of the participants have low- ASE, $61.2 \%$ medium-ASE, and the $21.4 \%$ high-ASE. Male participants reported more ASE than their peer females, and the 5th-year students have more ASE than 1st-year students.

Keywords: Academic self-efficacy, perceived self-efficacy, university students. 


\section{Introducción}

Desde que Bandura (1977) introdujo la teoría del aprendizaje social, la autoeficacia se convirtió en un constructo que ha ocupado un rol central en el trabajo de los psicólogos en su afán de comprender y predecir la conducta humana (Gore, 2006). La autoeficacia se define como un conjunto de creencias que un individuo desarrolla, con base en sus propias percepciones, con respecto a su capacidad para lograr con éxito sus objetivos personales (Bandura, 1977). Siguiendo esta línea de pensamiento, la autoeficacia académica (AA) puede ser entendida como la confianza en sí mismo que tiene un estudiante de su capacidad para lograr un exitoso desempeño en tareas académicas específicas (Schunk, 1991).

La aplicación de la teoría social cognitiva en el ámbito educativo permite realizar intervenciones con el objetivo de mejorar y optimizar el desempeño de los estudiantes, dado que la AA es un predictor importante del desempeño de los estudiantes, aunque el éxito logrado en tareas similares que hayan realizado en el pasado tendría mayor poder predictivo (Salanova, 2011).

Esto es, los estudiantes con altos niveles de AA y confianza en su desempeño académico probablemente crean que la inteligencia es variable y que se encuentra determinada por el esfuerzo, y al mismo tiempo prefieran metas académicas tales como buscar nuevos descubrimientos y conocimientos, conseguir nuevos grados, ser conocido por otros estudiantes y mostrar su inteligencia a través de su trabajo en la escuela (Komarraju y Nadler, 2013); en cambio, los estudiantes que tienen bajos niveles de AA y se encuentren inseguros con respecto a su éxito en los estudios tienen más probabilidad de creer que la inteligencia es una entidad fija e innata que no puede cambiar y, en consecuencia, tienen menos posibilidades de motivarse en relación con su rendimiento o logro de metas académicas, dado que consideran que su situación no cambiará (Komarraju y Nadler, 2013).

En cualquier caso, los estudiantes requieren una retroalimentación académica y social con respecto a su desempeño antes que ellos puedan realmente evaluar su capacidad para obtener logros académicos. En estas circunstancias, quienes mejor pueden hacer este trabajo son las personas encargadas de la labor tutorial, preferentemente al final del primer semestre o al inicio del segundo (Gore, 2006), que es el momento oportuno y crítico para promover las creencias de AA en estudiantes ingresados en su primer año, y seguir con este procedimiento a lo largo de la carrera a fin de lograr un balance entre las creencias de AA y las competencias reales.

En este sentido, es necesario conocer cómo se manifiesta la AA en la población universitaria, y sobre todo saber qué proporción de estudiantes posee baja AA, ya que esta situación representaría un serio obstáculo para la consecución de sus objetivos académicos a mediano y largo plazo.

A la fecha, no existen estudios de prevalencia relacionados con la AA en Perú. En otros contextos, el reporte de AA se efectúa en el marco del modelo de burnout (Maslach, Schaufeli, y Leiter, 2001) aplicado al ámbito universitario, y específicamente con el uso de la dimensión "Eficacia" del Maslach Burnout Inventory-Survey Student (MBI-SS) (Schaufeli, Martínez, Pinto, Salanova y Bakker, 2002; Schaufeli, Salanova, Gonzàlez-Romà y Bakker, 2002).

Dicha dimensión ha presentado algunos cambios que es conveniente resaltar. Si bien en un primer momento predominó un fraseo positivo de los ítems pertenecientes a "Eficacia" (cuya puntuación era revertida para el cálculo de la puntuación total del burnout), posteriormente se indicó que debido a la presencia de una relación más robusta con los otros dos constructos del modelo debe utilizarse directamente un fraseo negativo porque la experiencia de "Ineficacia" se asocia más al síndrome de burnout que la "Eficacia" (Bresó, Salanova y Schaufeli, 2007; Maroco, Maroco y Campos, 2014; Morgan, De Bruin y De Bruin, 2014; Schaufeli y Salanova, 2007). No obstante, existen algunos puntos que es necesario mencionar.

El primero de ellos es que ambas, "Eficacia" e "Ineficacia", no tienen una asociación completamente opuesta, es decir, su correlación es negativa, pero moderada (Bresó et al., 2007; Morgan et al., 2014; Schaufeli y Salanova, 2007), por lo que no serían intercambiables. 
El segundo es que la correlación de las puntuaciones de "Eficacia" es más fuerte con criterios externos, como las dimensiones del engagement, en comparación a las correlaciones observadas entre Ineficacia y esas mismas dimensiones (Schaufeli y Salanova, 2007). Inclusive la relación de ambas no difiere en intensidad con una (Morgan et al., 2014; Schaufeli y Salanova, 2007) o las otras dos dimensiones de burnout desde el modelo de Maslach (Bresó et al., 2007).

El tercero es que los coeficientes a calculados a partir de las puntuaciones de Ineficacia son a menudo de baja magnitud ( $a \leq .65$ ) (Bresó et al. 2007; Schaufeli y Salanova, 2007), por lo que sería arriesgado considerarlos en estudios de prevalencia debido a que el error de medición puede afectar las estimaciones (Zimmerman y Zumbo, 2015), concluyendo cifras que no reflejan adecuadamente lo que ocurre. En contraste, en la versión original (Schaufeli, Martinez et al., 2002; Schaufeli, Salanova et al., 2002) las magnitudes de los coeficientes de confiabilidad encontrados con Eficacia fueron al menos aceptables $(a>.70)$. A su vez, en otros estudios no existe una diferencia sustancial entre los coeficientes a de Eficacia e Ineficacia, siendo aceptables en ambos casos (a > .80) (Maroco et al., 2014; Morgan et al., 2014).

Esta situación se replica en otros trabajos empíricos en los que las magnitudes de los coeficientes a no son del todo satisfactorias para Eficacia (Atalayin, Balkis, Tezel, Onal y Kayrak, 2015; Hu y Schaufeli, 2009; Galán, Sanmartín, Polo y Giner, 2011), e incluso el coeficiente $\omega$ corregido por errores correlacionados (Dominguez, 2016) obtenido con un re-análisis ${ }^{1}$ fue de 697 (Tsubakita, y Shimazaki, 2016). No obstante, en otros reportes los coeficientes a fueron aceptables (> .80) (Rostami, Abedi, Schaufeli, Ahmadi y Sadeghi, 2013; Yavuz y Dogan, 2014; Zhang, Gan y Cham, 2007).

Con todo, la mayoría de los estudios usan la primera opción, es decir, evalúan Eficacia, y posteriormente invierten las puntuaciones, como se especifica en los escritos originales del MBI-ss (Schaufeli, Martinez et al., 2002; Schaufeli, Salanova et al., 2002).

Cuarto, y quizás el más relevante, es que no fue evaluado apropiadamente el efecto del método asociado a ítems invertidos (Lance, Dawson, Birkelbach y Hoffman, 2010) en el marco de la evaluación del burnout como constructo general. Es decir, no es posible saber si la variabilidad que aporta dicha dimensión, ya sea como Eficacia o Ineficacia, se debe en mayor grado al constructo o la forma como se califican los ítems. Si bien no es un procedimiento recurrente, es necesaria su implementación cuando son utilizados instrumentos con ítems invertidos (Dominguez-Lara y Merino-Soto, 2018).

\subsection{Prevalencia de baja autoeficacia académica}

Los estudios de prevalencia de baja AA no son numerosos porque, como se indicó anteriormente, quienes la abordan lo hacen en el marco del burnout bajo el modelo de Maslach y usando la dimensión Eficacia de su inventario, y en su mayoría sólo indican la prevalencia de este último en términos globales (Bolaños, y Rodríguez, 2016; Chigerwe, Boudreaux y llkiw, 2014; Fares et al., 2016; Mazurkiewicz, Korenstein, Fallar y Ripp, 2012; Ríos-Risquez, García-Izquierdo, Sabuco-Tebar, Carrillo-Garcia y Martinez-Roche, 2016), dejando de lado la prevalencia de cada dimensión de forma individual, incluida la AA.

No obstante, pese a que en algunos trabajos se reporta por separado también la prevalencia de baja AA se observan resultados divergentes. Por un lado, están aquellos donde la baja AA alcanza al $50 \%$ de estudiantes de diversas carreras (Kristanto, Chen y Thoo, 2016), u otros donde el 25\% de estudiantes de administración posee baja AA (Nikodijević, Labrović, y Doković, 2012), ambos estudios desarrollados con estudiantes de pregrado. Asimismo, investigaciones realizadas en estudiantes de posgrado brindan un 24.8\% de baja AA (Galdino, Martins, Haddad, Robazzi y Birolim, 2016).

En cambio, otras investigaciones brindan cifras diferentes incluso con grupos de estudiantes de la misma сагrera. En estudiantes de Medicina se han encontrado tasas de 17.8\% (Galán et al., 2011), 35\% (EstelaVilla, Jiménez-Román, Landeo-Gutiérrez, Tomateo-Torvisco, y Vega-Dienstmaier, 2010), e incluso del 52\%

\footnotetext{
${ }^{1}$ Para el cálculo fueron utilizados los datos publicados en Tsubakita y Shimazaki (2016).
} 
con baja AA (Chang, Eddins-Folensbee y Coverdale, 2012). Finalmente, se indica que $17.9 \%$ de estudiantes de Odontología (Atalayin et al., 2015) tienen baja AA. Existe un reporte con 0\% de baja AA en graduados de Enfermería (De Oliveira, Caregnato y Câmara, 2012). Es necesario precisar que pese a que Psicología es una carrera que implica el contacto directo con las personas y susceptible al síndrome de burnout, no fueron halladas investigaciones sobre prevalencia de burnout o AA.

Probablemente las diferencias halladas en torno a la AA obedezcan tanto a las diferencias culturales como a la metodología empleada para determinar los puntos de corte (PC) utilizados para clasificar a las personas. Por ejemplo, algunos estudios establecieron sus PC usando cuartiles o la desviación estándar (Atalayin et al., 2015; Galán et al., 2011; Nikodijević et al., 2012), otros utilizaron los PC del manual profesional (Galdino et al., 2016), mientras que en algunos estudios no informan qué referencia fue empleada (De Oliveira et al., 2012; Krisanto et al., 2016).

Además, y asumiendo que la diversidad de PC obedeció a criterios bien establecidos, en ningún estudio fue reportada la confiabilidad de los PC, aun cuando es un procedimiento necesario cuando se desea clasificar a las personas en determinado nivel de la variable evaluada (Dominguez-Lara, 2016a), como ocurre en los estudios del burnout (Fernández y Merino, 2014). Inclusive, si existieran valores normativos, los indicadores de confiabilidad encontrados en los reportes anteriores no parecen ser garantía de una adecuada clasificación.

\subsection{Escala de Autoeficacia Percibida Específica de Situaciones Académicas}

En este sentido, a fin de desarrollar un estudio que pueda recoger las características particulares del grupo estudiado con relación a la AA, es conveniente utilizar un instrumento que sea robusto, tanto en su estructura interna y la confiabilidad de sus puntuaciones, y poseer -además- datos normativos que permitan una adecuada clasificación de las personas, es decir, con PC confiables.

En este sentido, una opción para evaluar breve y unidimensionalmente la AA es la Escala de Autoeficacia Percibida Específica de Situaciones Académicas (EAPESA) (Palenzuela, 1983), la cual cuenta con diversos estudios instrumentales que garantizan sus propiedades psicométricas en diferentes contextos culturales, incluyendo el Perú (Dominguez-Lara, 2014a; Dominguez-Lara, Villegas, Yauri, Mattos y Ramírez, 2012; García-Fernández, Inglés, Torregosa et al., 2010; García-Fernández, Inglés, Vicent et al., 2016), además de poseer datos normativos adecuados para universitarios peruanos (Dominguez-Lara, 2016b). Un aspecto que la diferencia de la escala análoga del MBI-SS, es que la estimación de confiabilidad de esta última varía de un estudio a otro de forma notoria, mientras que la de la EAPESA se mantiene en niveles elevados en todos los estudios realizados. Por este motivo, la EAPESA parece la opción apropiada para cumplir con los objetivos del estudio debido a sus indicadores más robustos.

\subsection{El presente estudio}

De acuerdo con lo presentado, y lo relevante que es identificar estudiantes con baja AA para intervenir de forma eficiente (Mercer, Nellis, Martínez y Kirk, 2011), se plantea la necesidad de este estudio.

Asimismo, dado que la evidencia previa indica alguna relación de la autoeficacia con el sexo (Huang, 2013) es necesaria una valoración por separado. Por último, se propone un análisis y comparación por año de estudios debido a que a medida que el estudiante avanza en su vida académica se espera que adquiera una mayor experiencia y manejo de las actividades que debe llevar a cabo, incrementando sus experiencias de dominio (una de las fuente de autoeficacia) y, en consecuencia, su AA aumente a medida que avanza en la carrera profesional (Fogaça et al., 2012). No obstante, existe otro estudio que reporta similitudes en cuanto a AA entre estudiantes ingresantes y concluyentes (Tarnowski y Carlotto, 2007), pero la muestra fue pequeña ( $n=33$ por cada grupo) por lo que podría considerarse como un hallazgo provisional.

En tal sentido, el objetivo de este estudio fue investigar cuál es la prevalencia de baja AA en estudiantes universitarios de Psicología, así como analizar sus diferencias con relación al sexo y año de estudios. 


\section{Método}

El presente reporte descriptivo y transversal (Ato, López, y Benavente, 2013) contó con la participación de 730 estudiantes predominantemente de sexo femenino (76.6\% mujeres; Datos perdidos $=1$ ), $96.7 \%$ solteros, de 10. al 5o. año de estudios de la carrera de Psicología pertenecientes a una universidad privada ubicada en Lima Metropolitana (Perú). Las edades oscilaron entre 16 y 47 años ( $M=20.72$; DE = 3.525; Datos perdidos $=11$ ), siendo la edad de los varones, en promedio, mayor que la edad de las mujeres $(d=.24)$.

El instrumento empleado fue la versión adaptada al contexto universitario peruano de la Escala de Autoeficacia Percibida Específica de Situaciones Académicas (EAPESA) (Dominguez-Lara, 2014a; Dominguez-Lara et al., 2012), que consiste en una medida unidimensional formada por 9 ítems con 4 opciones de respuesta (de Nunca hasta Siempre). Las instrucciones piden responder con qué frecuencia manifiestan creencias vinculadas a su autoeficacia académica (AA). El puntaje total se halla mediante la suma aritmética de las puntuaciones de cada ítem.

Luego de obtener el permiso del decano de la facultad, los estudiantes resolvieron los cuestionarios en sus aulas, de manera voluntaria y anónima. Previamente, los investigadores dieron las instrucciones y condiciones de la recolección de datos en voz alta, permaneciendo en el aula durante el tiempo de aplicación; los estudiantes leyeron y firmaron el consentimiento informado para luego responder las preguntas en un ambiente de normalidad.

\subsection{Análisis estadístico}

Primero fueron analizadas las características descriptivas de las puntuaciones totales de la EAPESA (media, desviación estándar y coeficiente de variación) y distribucionales (asimetría y curtosis), así como la confiabilidad de las puntuaciones por medio del coeficiente a de Cronbach, agregando al reporte sus intervalos de confianza (IC) al 95\% (Dominguez-Lara, 2016c) calculados con el módulo ICalfa (DominguezLara y Merino-Soto, 2015). Dado que no fue realizado un análisis de tau-equivalencia, fue calculado el coeficiente a ordinal ( $a_{\text {ordinal }}$ ) (Elosua y Zumbo, 2008) con un módulo específico (Dominguez-Lara, 2012). Posteriormente fue calculado el índice de atenuación ( $\left[a-a_{\text {ordinal }}\right]$ / $a_{\text {ordinal }}$ ) que permite cuantificar la infraestimación del coeficiente en presencia de una indicador más preciso, como el $a_{\text {ordinal }}$ (Zumbo, Gadermann y Guhn, 2007).

Si bien es recomendable el análisis de invarianza de medición en casos como este (Lee, Puig, Lea y Lee, 2013), pero en vista que para ejecutar este tipo de análisis es recomendable al menos 200 individuos de cada grupo (Dimitrov, 2010), fueron implementados dos procedimientos con el objetivo de evaluar la equivalencia entre grupos:

El primero fue un análisis de congruencia factorial de la EAPESA entre los subgrupos de cada variable de comparación (sexo y año de estudios). Previamente, se realizó un análisis factorial exploratorio con el programa FACTOR (Lorenzo-Seva y Ferrando, 2013). El método de extracción factorial fue el de mínimos cuadrados no ponderados con base en una matriz de correlaciones policóricas (Olsson, 1979a, 1979b) dado que los ítems son variables ordinales (Dominguez-Lara, 2014b), y forzando la extracción a un solo factor. Posteriormente, las estructuras factoriales fueron comparadas utilizando el Coeficiente de Congruencia $(J)$ (García-Cueto, 1994) que fue calculado con un módulo especializado (Dominguez-Lara, 2013). Si el valor de $J$ es estadísticamente significativo $(p<.05)$ puede concluirse que las dos estructuras comparadas son similares. Para llevar a cabo este análisis fue tomada como estructura referente la hallada en los estudiantes de 20. gardo que poseen el mayor tamaño muestral.

El segundo fue la comparación estadística de los coeficientes a obtenidos, tanto según el sexo como el año de estudios, mediante un método asintótico basado en la distribución $F$ (Feldt, 1980; Feldt, Woodruff y Salih, 1987) utilizando el programa AlphaTest (Lautenschlager y Meade, 2008). El objetivo fue conocer la equivalencia en cuanto a la estimación del error de medición (Merino y Lautenschlager, 2003). 
En cuanto al análisis principal, la prevalencia fue determinada usando los puntos de corte provistos en un trabajo normativo preliminar (Dominguez-Lara, 2016b). De acuerdo con éste, las puntuaciones mayores que 31 indican alta AA, y menores de 23, baja AA. Estos resultados fueron reportados tanto por sexo como por año de estudios; después de esto fueron comparadas las proporciones observadas entre los estudiantes según el sexo, y entre estudiantes de 10. y 5o. año, utilizando el $h$ de Cohen (Cohen, 1992) que se valora como insignificante (<.20), pequeña (entre .20 y .50), mediana (entre .50 y .80), o grande (> $.80)$.

Del mismo modo, las puntuaciones brutas de AA de hombres y mujeres fueron comparadas utilizando la $d$ de Cohen (Cohen, 1992). La d fue valorada en los mismos términos que el estadístico anterior: insignificante $(<.20)$, pequeña (entre .20 y .50), mediana (entre .50 y .80), o grande ( $>.80$ ); y las puntuaciones brutas de AA de los estudiantes de 10. a 50. año fueron comparadas por medio de una medida de magnitud del efecto basada en el análisis de varianza (Anova) tanto a nivel general $\left(\omega^{2}\right)$ como en comparaciones pareadas ( $\omega^{2}$ comp) (Dominguez-Lara, 2017). Ambos coeficientes fueron valorados de la siguiente forma: insignificante (<.04); mínima (entre .04 y .25), moderada (entre .25 y .64) y fuerte (> .64) (Fritz, Morris y Richler, 2012).

\section{Resultados}

El promedio de respuesta de la EAPESA fue elevado $(M=27.200)$ y con una dispersión que refleja homogeneidad ( $D E=5.028 ; C V=.185)$. A su vez, los estadísticos de asimetría $\left(g_{1}=-.118\right)$ y curtosis $\left(g_{2}=-.585\right)$ fueron de baja magnitud. Por último, el análisis de la confiabilidad mostró indicadores elevados en la muestra global ( $a=.905$, IC95\% .891, .917).

En cuanto al análisis factorial realizado, se aprecia una estructura interna robusta en todos los casos (KMO > .890; porcentaje de varianza explicada > 50\%) (ver tabla I). Asimismo, el coeficiente de congruencia fue de .999 ( $p$ < .001) entre hombres y mujeres, lo que indica que la estructura interna de la EAPESA es similar entre ambos grupos. Por otro lado, tomando como referencia la estructura factorial del segundo año (es la de mayor muestra) sus coeficientes de congruencia generales con sus pares de 10., 30., 40. y 50. año fueron de .999, .997, .998, y .997, respectivamente, todos ellos estadísticamente significativos ( $p<.001)$.

Tabla I. Estructura factorial de la EAPESA según sexo y año de estudios

\begin{tabular}{|c|c|c|c|c|c|c|c|}
\hline & \multicolumn{2}{|c|}{ Sexo } & \multicolumn{5}{|c|}{ Año de estudios } \\
\hline & Hombres & Mujeres & 10. & 20. & 30. & 40. & 50. \\
\hline Ítem 1 & .733 & .787 & .722 & .796 & .736 & .728 & .709 \\
\hline Ítem 2 & .754 & .765 & .697 & .765 & .746 & .781 & .708 \\
\hline Ítem 3 & .792 & .803 & .716 & .799. & .768 & .808 & .759 \\
\hline Ítem 4 & .779 & .770 & 699 & .771 & .784 & .748 & .770 \\
\hline Ítem 5 & .771 & .774 & .762 & .802 & .674 & .740 & .700 \\
\hline Ítem 6 & .778 & .833 & .732 & .816 & .737 & .787 & .855 \\
\hline Ítem 7 & .714 & .677 & .607 & .660 & .630 & .745 & .748 \\
\hline Ítem 8 & .626 & .722 & .694 & .657 & .701 & .673 & .728 \\
\hline Ítem 9 & .793 & .804 & .805 & .794 & .676 & .747 & .776 \\
\hline KMO & .921 & .930 & .920 & .920 & .906 & .920 & .899 \\
\hline $\begin{array}{l}\text { \% varianza } \\
\text { explicada }\end{array}$ & 61.069 & 63.996 & 56.666 & 62.913 & 56.925 & 61.262 & 61.260 \\
\hline
\end{tabular}

Asimismo, las magnitudes del coeficiente a y aordinal alcanzaron niveles elevados (>. 89), y los índices de atenuación fueron cercanos a cero. Por otro lado, los coeficientes a fueron similares de acuerdo al sexo y año de estudios, como se ilustra en la tabla II. 
Tabla II. Coeficientes de confiabilidad y diferencias según sexo y año de estudios

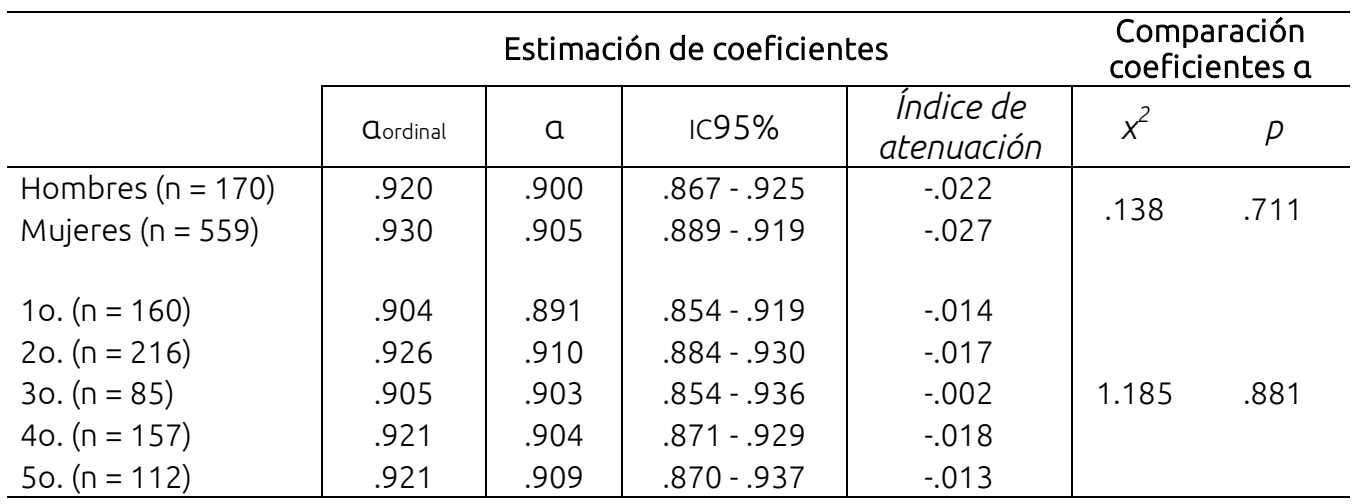

De acuerdo con los cálculos realizados, el 17.4\% (IC95\% 14.84 - 20.34\%) presenta baja AA; el 61.2\% (IC95\% 57.59 - 64.65) AA promedio; y un $21.4 \%$ (IC95\% 18.58 - 24.52\%) reportan altos niveles de AA.

En cuanto a la comparación entre hombres y mujeres, las mujeres evidencian mayor proporción de niveles bajos de AA y menor proporción de niveles altos de AA. Por otro lado, con relación a la comparación entre estudiantes de 10. y 5o. año se aprecia que a medida que se avanza en los años de estudio se incrementa el número de personas que posee un nivel alto de AA y una reducción de estudiantes con un nivel bajo de AA (ver tabla III).

Tabla III. Niveles de autoeficacia académica: prevalencia según sexo y año de estudios

\begin{tabular}{lcccccc}
\hline & \multicolumn{3}{c}{ Nivel de autoeficacia académica } \\
\cline { 2 - 7 } & \multicolumn{3}{c}{ Bajo } & \multicolumn{2}{c}{ Medio } & \multicolumn{2}{c}{ Alto } \\
\cline { 2 - 7 } & $p$ & $h$ & $p$ & $h$ & $p$ & $h$ \\
\hline Hombres $(n=170)$ & .124 & .182 & .588 & .063 & .288 & .228 \\
Mujeres $(n=559)$ & .190 & & .619 & & .191 & \\
& & & & & & \\
10. $(n=160)$ & .225 & & .625 & & .150 & \\
20. $(n=216)$ & .222 & & .574 & & .204 & \\
3o. $(n=85)$ & .141 & $.352^{\text {a }}$ & .624 & $.019^{a}$ & .235 & $.293^{\text {a }}$ \\
4o. $(n=157)$ & .127 & & .624 & & .248 & \\
50. $(n=112)$ & .098 & & .634 & & .268 & \\
\hline
\end{tabular}

Nota: $p$ : proporción con referencia a su grupo de pertenencia (e.g., varones); $h$ : magnitud del efecto para diferencia de proporciones; $a$ : comparación realizada entre estudiantes de primer y último año.

Finalmente, los hombres presentan un mayor promedio que las mujeres en AA $(d=.32)$; y una comparación conjunta de los estudiantes de 10. al 5o. año en cuanto a las puntuaciones brutas de AA indica que las diferencias no fueron significativas $\left(\omega^{2}=.016\right)$ ni en cuanto a las comparaciones post-hoc por pares $\left(\omega_{\text {comp }}^{2}<.02\right)$

\section{Discusión}

El objetivo principal de esta investigación fue conocer cómo se manifiesta la AA en estudiantes universitarios de Psicología y analizar las diferencias existentes con relación al sexo y año de estudios.

En primer lugar, la prevalencia de baja AA hallada es similar a lo reportado con estudiantes de Medicina (17.8\%) (Galán et al., 2011) y Odontología (17.9\%) (Atalayin et al., 2015), pero dista de lo encontrado en grupos heterogéneos de estudiantes (Kristanto et al., 2016). 
Esto indicaría que sería mejor analizar la manifestación de la AA en cada carrera por separado, ya que de ese modo las conclusiones podrían ser más realistas; es decir, podrían circunscribirse a un determinado contexto académico porque cada carrera profesional posee: a) un grado de exigencia que, hasta cierto punto, podría incidir en la AA percibida por el estudiante, b) un perfil particular del estudiante, cuyas características de personalidad podrían estar involucradas también. Asimismo, el instrumento de medición utilizado es distinyo entre los estudios, por lo que las diferencias, hasta cierto punto, podrían ser atribuidas a ello.

Por otro lado, con relación al sexo fueron halladas diferencias en AA a favor de los hombres, lo que va en consonancia con investigaciones anteriores (Huang, 2013). Esto quiere decir que los estudiantes hombres se perciben con mayores recursos que sus pares de sexo femenino.

Respecto al año de estudios, se observa que la diferencia de AA entre los diferentes grupos no fue significativa considerando un análisis conjunto. Aquello podría llevar a la conclusión de que la AA percibida no aumenta ni decrece según el avance en la carrera universitaria. Sin embargo, cuando son considerados sólo los estudiantes de 10. y 5o. año se aprecia que estos últimos poseen una mayor proporción de estudiantes con alta AA. Es decir, el aumento gradual de personas con alta AA y disminución de personas con baja AA a medida que avanzan los años de estudio indicaría que el cambio no es lo suficientemente notorio año tras año como para considerarse importante, pero sí indicaría una mejora significativa a mediano plazo.

Esto probablemente se debe a que en los últimos años la mayoría de los estudiantes realiza prácticas preprofesionales no oficiales que les brindan, además de un refuerzo al conocimiento que ya adquirieron, la oportunidad de experimentar directamente labores relacionadas con la carrera, reforzando y fortaleciendo de ese modo sus creencias de AA. Asimismo, los estudiantes de quinto año se encuentran realizando prácticas pre-profesionales oficiales como parte del plan curricular conducente al título profesional, y dentro de ese período deben cursar materias relacionadas con la especialidad escogida (por ejemplo, Psicología educativa), además de revisar y exponer materiales bibliográficos en la institución donde llevan a cabo dichas prácticas.

Estos resultados podrían alentar a que los docentes planifiquen los sílabos y diseñen las actividades en el aula de clases con el objetivo de desarrollar la AA en los estudiantes incorporando, de manera estratégica, las cuatro fuentes de eficacia de Bandura (1977) aplicadas a la labor del psicólogo: experiencias exitosas, modelación, persuasión social y manejo del estado fisiológico. Por ejemplo, podrían brindar a los estudiantes oportunidades de tener experiencias de éxito mediante tareas de complejidad creciente; observar modelos con alto nivel de logros a través de conferencias de psicólogos destacados; o ensayar estrategias para manejar obstáculos y practicar tácticas para manejar la ansiedad en el desempeño con evaluaciones supervisadas (Komarraju y Nadler, 2013).

De igual forma, los instructores podrían enfatizar el valor práctico de los materiales que están siendo aprendidos y la importancia del esfuerzo, lo que podría fortalecer la motivación del estudiante (Zusho y Pintrich, 2003). También podría proporcionarles ejemplos claros de trabajo académico clarificando expectativas y dando amplia retroalimentación para ayudar a los estudiantes a desarrollar la AA (Lane y Lane, 2001). Si bien estas labores son incorporadas rutinariamente por los docentes en las asignaturas, no siempre son realizadas bajo un marco teórico organizado y no se evalúa la eficacia de dicha implementación, por lo que sería oportuno ejecutar y evaluar dichas acciones.

Aunque se trató de contar con una muestra lo suficientemente amplia, ésta no es representativa de toda la población universitaria. Por ello, los resultados sólo son aplicables a la carrera e institución participantes y, aunque con prudencia, a universidades que compartan sus características (privada, predominantemente de nivel socioeconómico medio, etc.). Si bien la proporción entre hombres y mujeres puede causar preocupación, la proporción observada es la común en investigaciones realizadas en estudiantes de Psicología, además de que los análisis preliminares indican que no existe sesgo en la medida del constructo. Por otro lado, si bien fue realizado un análisis con todos los años de estudio, esto no reemplaza un estudio longitudinal, donde se puede apreciar el grado de variabilidad que experimenta 
la AA de un mismo estudiante a lo largo de los años de estudios.

En conclusión, la prevalencia hallada indica que uno de cada cinco estudiantes de Psicología posee baja AA, lo que podría colocarlo en riesgo dado los correlatos observados de la AA con variables que merman el desempeño académico (por ejemplo: agotamiento emocional), ante lo cual es recomendable tomar acciones inmediatas por parte de los encargados de la atención al estudiante.

\section{Referencias}

Atalayin, C., Balkis, M., Tezel, H., Onal, B. y Kayrak, G. (2015). The prevalence and consequences of burnout on a group of preclinical dental students. European Journal of Dentistry, 9(3), 356-363. doi:10.4103/13057456.163227

Ato, M., López, J. y Benavente, A. (2013). Un sistema de clasificación de los diseños de investigación en psicología. Anales de Psicología, 29(3), 1038-1059. doi:10.6018/analesps.29.3.178511

Bandura, A. (1977). Self-efficacy: Toward unifying theory of behavior change. Psychological Review, 84, 191-215. doi:10.1037/0033-295X.84.2.191

Bolaños, N. y Rodríguez, N. (2016). Prevalencia del Síndrome de Burnout académico en el estudiantado de Enfermería de la Universidad de Costa Rica. Enfermería Actual de Costa Rica, 31. Recuperado de http://revistas.ucr.ac.cr/index.php/enfermeria/article/download/24519/25894

Bresó, E., Salanova, M., y Schaufeli, W. B. (2007). In search of the "third dimension" of burnout: Efficacy or inefficacy? Applied Psychology, 56(3), 460-478. doi:10.1111/j.1464-0597.2007.00290.x

Chang, E., Eddins-Folensbee, F. y Coverdale, J. (2012). Survey of the prevalence of burnout, stress, depression, and the use of supports by medical students at one school. Academic Psychiatry, 36(3), 177182. doi:10.1176/appi.ap.11040079

Chigerwe, M., Boudreaux, K. A., y llkiw, J. E. (2014). Assessment of burnout in veterinary medical students using the Maslach Burnout Inventory-Educational Survey: a survey during two semesters. BMC Medical Education, 14(1). Recuperado de https://bmcmededuc.biomedcentral.com/articles/10.1186/s12909-014$\underline{0255-4}$

Cohen, J. (1992). A power primer. Psychological Bulletin, 112(1), 155-159.

De Oliveira, R., Caregnato, R. C. A. y Câmara, S. G. (2012). Síndrome de Burnout em acadêmicos do último ano da graduação em enfermagem [Síndrome de Burnout en estudiantes de pregrado de enfermería]. Acta Paulista de Enfermagem, 25(2), 54-60. doi:10.1590/0104-1169.3254.2498

Dimitrov, D. M. (2010). Testing for factorial invariance in the context of construct validation. Measurement and Evaluation in Counseling and Development, 43(2), 121-149. doi:10.1177/0748175610373459

Dominguez, S. (2012). Propuesta para el cálculo del Alfa Ordinal y Theta de Armor. Revista de Investigación en Psicología, 15(1), 213-217. doi:10.15381/rinvp.v15i1.3684

Dominguez, S. (2013). Coeficiente de congruencia: propuesta de cálculo. Pensando Psicología, 9(16), 107109. Recuperado de https://www.scribd.com/document/223977892/Coeficiente-de-congruencia-propues ta-de-calculo

Dominguez, S. (2014a). Autoeficacia para situaciones académicas en estudiantes universitarios peruanos: un enfoque de ecuaciones estructurales. Revista de Psicología, 4, 45-53. Recuperado de http://ucsp.edu.pe/investigacion/psicologia/wp-content/uploads/2015/03/Autoeficacia-para-situacionesacademicas.pdf 
Dominguez, S. (2014b). ¿Matrices policóricas/tetracóricas o matrices Pearson? Un estudio metodológico. Revista Argentina de Ciencias del Comportamiento, 6(1), 39-48. Recuperado de https://revistas.unc.edu.ar/index.php/racc/article/view/6357

Dominguez, S. (2016). Errores correlacionados y estimación de la fiabilidad en estudios de validación: comentarios al trabajo validación de la escala ehealth literacy (eheals) en población universitaria española. Revista Española de Salud Pública, 90(9), e1-e2.

Dominguez, S., Villegas, G., Yauri, C., Mattos, E. y Ramírez, F. (2012). Propiedades psicométricas de una escala de autoeficacia para situaciones académicas en estudiantes universitarios peruanos. Revista de Psicología-UCSP, 2(1), 27-39.

Dominguez-Lara, S. (2016a). Confiabilidad de los puntos de corte en estudios empíricos. Revista Cubana de Pediatría, 88(4). Recuperado de http://www.revpediatria.sld.cu/index.php/ped/article/view/88/75

Dominguez-Lara, S. (2016b). Valores normativos de una escala de autoeficacia académica en estudiantes universitarios de Lima. Interacciones, 2(2), 91-98. doi:10.24016/2016.v2n2.31

Dominguez-Lara, S. (2016c). Intervalos de confianza en el reporte de la fiabilidad: un análisis necesario [Carta al editor]. Anales del Sistema Sanitario de Navarra, 39(1), pp. 169-170.

Dominguez-Lara, S. (2017). Magnitud del efecto en comparaciones entre dos o más grupos. Revista de Calidad Asistencial, 32(2), 121-122.

Dominguez-Lara, S. y Merino-Soto, C. (2015) ¿Por qué es importante reportar los intervalos de confianza del coeficiente alfa de Cronbach? Revista Latinoamericana de Ciencias Sociales, Niñez y Juventud, 13(2), 1326-1328.

Dominguez-Lara, S. y Merino-Soto, C. (2018). Efectos de método en el Inventario de Depresión EstadoRasgo (IDER): un análisis SEM. Avances en Psicología Latinoamericana, 36(2), 253-267.

doi:10.12804/revistas.urosario.edu.co/apl/a.4151

Elosua, P. y Zumbo, B. D. (2008). Coeficientes de fiabilidad para escalas de respuesta categórica ordenada. Psicothema, 20(4), 896-901. Recuperado de http://www.psicothema.com/psicothema.asp? $\underline{\mathrm{id}=3572}$

Estela-Villa, L. M., Jiménez-Román, C. R., Landeo-Gutiérrez, J. S., Tomateo-Torvisco, J. D. y VegaDienstmaier, J. M. (2010). Prevalencia de síndrome de burnout en alumnos del séptimo año de medicina de una universidad privada de Lima, Perú. Revista de Neuropsiquiatría, 73(4), 147-156.

Fares, J., Saadeddin, Z., Al Tabosh, H., Aridi, H., El Mouhayyar, C., Koleilat, M. K.,Chaaya, M. y El Asmar, K. (2016). Extracurricular activities associated with stress and burnout in preclinical medical students. Journal of Epidemiology and Global Health, 6(3), 177-185. doi:10.1016/j.jegh.2015.10.003

Feldt, L. S. (1980). A test of the hypothesis that Cronbach's alpha reliability coefficient is the same for two tests administered to the same sample. Psychometrika, 45, 99-105. doi:10.1007/BF02293600

Feldt, L. S., Woodruff, D. J. y Salih, F. A. (1987) Statistical inference for coefficient alpha. Applied Psychological Measurement, 11, 93-103. doi:10.1177/014662168701100107

Fernández, M. y Merino, C. (2014). Error de medición alrededor de los puntos de corte en el MBI-GS. Liberabit, 20(2), 209-218. Recuperado de http://www.scielo.org.pe/pdf/liber/v20n2/a02v20n2.pdf

Fogaça, M. C., Hamasaki, E. I. M, Barbieri C. A. P., Borsetti, J., Martins, R. Z., Silva, I. G. y Ribeiro, L. P. (2012). Burnout em estudantes de psicologia: diferenças entre alunos iniciantes e concluintes [Burnout en 
estudiantes de psicología: diferencias entre estudiantes principiantes y graduados]. Aletheia, (38-39), 124131. Recuperado de http://pepsic.bvsalud.org/scielo.php?script=sci arttext\&pid=S1413-03942012000 200010\&lng=pt\&thng=pt

Fritz, C. O., Morris, P. E. y Richler, J. J. (2012). Effect size estimates: current use, calculations, and interpretation. Journal of Experimental Psychology: General, 141, 2-18.

Galán, F., Sanmartín, A., Polo, J. y Giner, L. (2011). Burnout risk in medical students in Spain using the Maslach Burnout Inventory-Student Survey. International Archives of Occupational and Environmental Health, 84(4), 453-459. doi:10.1007/s00420-011-0623-x

Galdino, M. J. Q., Martins, J. T., Haddad, M. C. F. L., Robazzi, M. L. C. C. y Birolim, M. M. (2016). Síndrome de Burnout entre mestrandos e doutorandos em enfermagem [Síndrome de burnout entre estudiantes de maestría y doctorado en Enfermería]. Acta Paulista de Enfermagem, 29(1), 100-106. doi:10.1590/19820194201600014

García Fernández, J., Inglés, C., Vicent, M., Gonzálvez, C., Pérez y San Martín, N. (2016). Validación de la Escala de Autoeficacia Percibida Específica de Situaciones Académicas en Chile y su relación con las estrategias de aprendizaje. Revista Iberoamericana de Diagnóstico y Evaluación-e Avaliação Psicológica, 41(1), 118-131. Recuperado de http://www.aidep.org/sites/default/files/articles/R41/Art10.pdf

García-Cueto, E. (1994). Coeficiente de congruencia. Psicothema, 6(3), 465-468. Recuperado de http://www.psicothema.com/psicothema.asp?id=940

García-Fernández, J., Inglés, C., Torregrosa, M., Ruiz, C., Díaz, A., Pérez, E. y Martínez, M. (2010). Propiedades psicométricas de la Escala de Autoeficacia Percibida Específica de Situaciones Académicas en una muestra de estudiantes españoles de Educación Secundaria Obligatoria. European Journal of Education y Psychology, 3(1), 61-74.

Gore, P. (2006). Academic Self-Efficacy as a Predictor of College Outcomes: Two Incremental Validity Studies. Journal of Career Assesment, 14(1), 92-115. doi:10.1177/1069072705281367

Hu, Q. y Schaufeli, W. B. (2009). The factorial validity of the Maslach Burnout Inventory-Student Survey in China. Psychological Reports, 105(2), 394-408. doi:10.2466/PR0.105.2.394-408

Huang, C. (2013). Gender differences in academic self-efficacy: a meta-analysis. European Journal of Psychology of Education, 28(1), 1-35. doi:10.1007/s10212-011-0097-y

Komarraju, M. y Nadler, D. (2013). Self-efficacy and academic achievement: Why do implicit beliefs, goals, and effort regulation matter? Learning and Individual Differences, 25, 67-72.

doi:10.1016/j.lindif.2013.01.005

Kristanto, T., Chen, W. S. y Thoo, Y. Y. (2016). Academic burnout and eating disorder among students in Monash University Malaysia. Eating behaviors, 22, 96-100. doi:10.1016/j.eatbeh.2016.03.029

Lance, C., Dawson, B., Birkelbach, D. y Hoffman, B. (2010). Method effects, measurement error, and substantive conclusions. Organizational Research Methods, 13(3), 435-455.

doi:10.1177/1094428109352528

Lane, A. y Lane, L. (2001). Self-efficay and academic performance. Social behavior and Personality: An International Journal, 29, 687-693. doi:10.2224/sbp.2001.29.7.687 
Lautenschlager, G. J. y Meade, A. W. (2008). AlphaTest: A windows program for tests of hypotheses about coefficient alpha. Applied Psychological Measurement, 23, 502-503. doi:10.1177/0146621607312307

Lee, J., Puig, A., Lea, E. y Lee, S. M. (2013). Age-related differences in academic burnout of Korean adolescents. Psychology in the Schools, 50(10), 1015-1031. doi:10.3923/jas.2015.661.667

Lorenzo-Seva, U. y Ferrando, P.J. (2013). FACTOR 9.2 A comprehensive program for fitting exploratory and semiconfirmatory factor analysis and IRT models. Applied Psychological Measurement, 37(6), 497-498. doi:10.1177/0146621613487794

Maroco, J., Maroco, A. L. y Campos, J. A. D. B. (2014). Student's academic efficacy or inefficacy? An example on how to evaluate the psychometric properties of a measuring instrument and evaluate the effects of item wording. Open Journal of Statistics, 4, 484 - 493. doi:10.4236/ojs.2014.46046

Maslach, C., Schaufeli, W. B., y Leiter, M. P. (2001). Job burnout. Annual Review of Psychology, 52, 397-422. doi:10.1146/annurev.psych.52.1.397

Mazurkiewicz, R., Korenstein, D., Fallar, R. y Ripp, J. (2012). The prevalence and correlations of medical student burnout in the pre-clinical years: a cross-sectional study. Psychology, Health y Medicine, 17(2), 188195. doi:10.1080/13548506.2011.597770

Mercer, S. H., Nellis, L. M., Martínez, R. S. y Kirk, M. (2011). Supporting the students most in need: academic self-efficacy and perceived teacher support in relation to within-year academic growth. Journal of School Psychology, 49, 323-338. doi:10.1016/j.jsp.2011.03.006

Merino, C., y Lautenschlager, G. (2003). Comparación estadística de la confiabilidad alfa de Cronbach: aplicaciones en la medición educacional. Revista de Psicología, 12, 129-139.

doi:10.5354/0719-0581.2012.17668

Morgan, B., De Bruin, G. P. y De Bruin, K. (2014). Operationalizing burnout in the Maslach Burnout Inventory-Student Survey: personal efficacy versus personal inefficacy. South African Journal of Psychology, 44(2), 216-227. doi:10.1177/0081246314528834

Nikodijević, A., Andelković, J. A. y Doković, A. (2012). Academic burnout among students at faculty of organizational sciences. XIII Simposio SymOrg 2012: Innovative management and business performance, Zlatibor, Serbia.

Olsson, U. (1979a). Maximum likelihood estimation of the polychoric correlation coefficient. Psychometrika, 44, 443-460. doi:10.1007/BF02296207

Olsson, U. (1979b). On the robustness of factor analysis against crude classification of the observations. Multivariate Behavioral Research, 14, 485-500. doi:10.1007/BF02296207

Palenzuela, D. (1983). Construcción y validación de una escala de autoeficacia percibida específica de situaciones académicas. Análisis y Modificación de Conducta, 9(21), 185- 219.

Ríos-Risquez, M. I., García-Izquierdo, M., Sabuco-Tebar, E. A., Carrillo-Garcia, C. y Martinez-Roche, M. E. (2016). An exploratory study of the relationship between resilience, academic burnout and psychological health in nursing students. Contemporary Nurse, 52(4), 430-439. doi:10.1080/10376178.2016.1213648

Rostami, Z., Reza Abedi, M., Schaufeli, W., Ahmadi, A. y Sadeghi, A. H. (2013). The psychometric characteristics of Maslach Burnout Inventory student survey: Among students of Isfahan University. Zahedan Journal of Research in Medical Sciences, 15, 29-32. Recuperado de

https://core.ac.uk/download/pdf/34626927.pdf 
Salanova, M. (2011). Psicología de la salud ocupacional. Madrid: Síntesis.

Schaufeli, W. B., Martinez, I. M., Pinto, A. M., Salanova, M. y Bakker, A. B. (2002). Burnout and engagement in university students a cross-national study. Journal of Cross-Cultural Psychology, 33(5), 464-481.

doi:10.1177/0022022102033005003

Schaufeli, W. B., Salanova, M., Gonzàlez-Romà, V. y Bakker, A. B. (2002). The measurement of burnout and engagement: A two simple confirmative analytic approach. Journal of Happiness Studies, 3, 71-92. doi: 10.1023/A:1015630930326

Schaufeli, W. B. y Salanova, M. (2007). Efficacy or inefficacy, that's the question: burnout and work engagement and their relationship with efficacy beliefs. Anxiety, Stress, y Coping, 20, 177-196.

doi:10.1080/10615800701217878

Schunk, D. H. (1991). Self-efficacy and academic motivation. Educational Psychology, 26, 207-231. doi: 10.1080/00461520.1991.9653133

Tarnowski, M. y Carlotto, M. S. (2007). Síndrome de Burnout em estudantes de psicología [Síndrome de burnout en estudiantes de Psicología]. Temas em Psicología, 15(2), 173-180.

Tsubakita, T., y Shimazaki, K. (2016). Constructing the Japanese version of the Maslach Burnout InventoryStudent Survey: Confirmatory factor analysis. Japan Journal of Nursing Science, 13(1), 183-188.

doi:10.1111/jjns.12082

Yavuz, G. y Dogan, N. (2014). Maslach Burnout Inventory-Student Survey (MBI-SS): A validity study. Procedia-Social and Behavioral Sciences, 116, 2453-2457. doi:10.1016/j.sbspro.2014.01.590

Zhang, Y., Gan, Y. y Cham, H. (2007). Perfectionism, academic burnout and engagement among Chinese college students: a structural equation modeling analysis. Personality and Individual Differences, 43(6), 1529-1540. doi:10.1016/j.paid.2007.04.010

Zimmerman, D. W. y Zumbo, B. D. (2015). Resolving the issue of how reliability is related to statistical power: adhering to mathematical definitions. Journal of Modern Applied Statistical Methods, 14(2), 9-26. doi:10.22237/jmasm/1446350640

Zumbo, B. D., Gadermann, A. M. y Guhn, M. (2007). Ordinal versions of coefficients alpha and theta for Likert rating scales. Journal of Modern Applied Statistical Methods, 6(1). doi:10.22237/jmasm/1177992180

Zusho, Z. y Pintrich, P. (2003). Skill and will: the rol of motivation and cognition in the learning of college chemistry. International Journal of Science Education, 25, 1081-1094. doi:10.1080/0950069032000052207 